Proc. XIX International School of Semiconducting Compounds, Jaszowiec 1990

\title{
DIFFUSION LENGTH STUDIES IN CdMnTe BY THE SURFACE PHOTOVOLTAGE METHOD*
}

\author{
E. Placzek-Popko, L. Szaro and L. Jędral \\ Institute of Physics, Technical University of Wroclaw, Wybrzeże Wyspiańskiego 27, \\ 50-370 Wroclaw, Poland \\ (Received August 8, 1990)
}

\begin{abstract}
The surface phototovoltage method provides a nondestructive means of measuring minority carriers diffusion length and is suitable for process control applications and for material acceptance tests. Application of the method in the case of CdMnTe compounds has been studied in the present paper. The optimum measurement conditions have been investigated by studying the dependence of measured diffusion length on the experimental conditions. As the surface photovoltage method requires the exact values of absorption coefficient as a function of wavelength, $\alpha=f(\lambda)$, the dependence has been determined. The minority carrier diffusion length for the sample investigated has been found to be equal to several tenth of $\mu \mathrm{m}$.
\end{abstract}

PACS numbers: $72.40 .+w$

The surface photovoltage is the change in the surface barrier height, $\Delta V_{\mathrm{sp}}$, produced by illumination of the free semiconductor surface. If the low injection condition is fulfilled, (i.e. when $\Delta V_{\mathrm{sp}}<k T / 2 e=12 \mathrm{meV}$ at $T=300 \mathrm{~K} \mathrm{[1]}$ ), the surface photovoltage, $\Delta V_{\mathrm{sp}}$, is the monotonic function of the excess carrier density injected at the surface [2] $\Delta V_{\mathrm{sp}}=f(\Delta n)$. Having neglected multiple reflections, it can be shown [2] that

$$
\Delta n=\frac{\eta I_{0}(1-R) \alpha L_{D}}{\left(D / L_{D}+s\right)\left(1+\alpha L_{D}\right)}
$$

where $\eta$ is the quantum efficiency, $I_{0}$ - light intensity, $R$ - reflection coefficient, $D$ - minority carrier diffusion coefficient, $s$ - surface recombination velocity, $\alpha$ - optical absorption coefficient, $L_{D}$ - minority carrier diffusion length. If we also neglect small variations of $\eta$ and $R$ with wavelength, $\lambda$, and assume that $d \gg L_{D}$, $d>a^{-1}, t \ll L_{D}$ (where $d$ is the sample thickness and $t$ is the space charge layer

*This work was supported by CPBP 01.08. E3.2. 
thickness), then the incident light intensity $I_{0}$ required to produce constant $\Delta V_{\mathrm{sp}}$ is a linear function of the reciprocal absorption coefficient, $\alpha^{-1}$ :

$$
I_{0} \approx c\left(\alpha^{-1}+L_{D}\right)
$$

where $c$ is a constant, independent of $\lambda$. The linear plot of $I_{0}=f\left(\alpha^{-1}\right)$ is then extrapolated to zero light intensity and negative intercept value is the effective minority carrier diffusion length $L_{D}$.

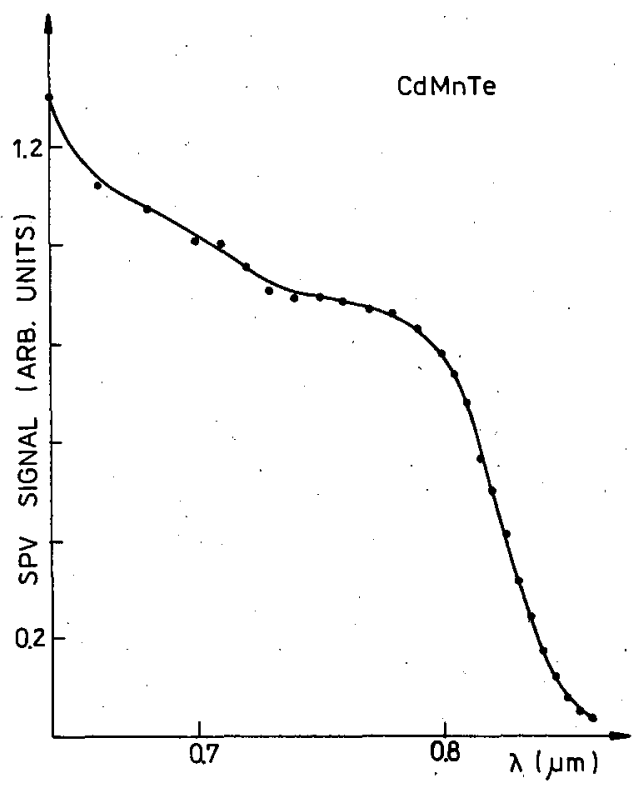

Fig. 1. Spectral characteristic of surface photovoltage, $\Delta V_{\mathrm{sp}}=f(\lambda)$ for a wide-gap $\left(E_{\mathrm{g}}=1.47 \mathrm{eV}\right.$ at $\left.300 \mathrm{~K}\right) \mathrm{CdMnTe}$.

The $p$-type CdMnTe samples (with energy gap $E_{\mathrm{g}}=1.47 \mathrm{eV}$ at $T=300 \mathrm{~K}$ ) were purchased from IF PAN Warsaw. The surface photovoltage measurements were performed as follows. Monochromatic light chopped with $12 \mathrm{~Hz}$ frequency illuminated surface of the specimen capacitatively coupled into the phase sensitive lock-in amplifier. Figure 1 shows spectral dependence of $\Delta V_{\mathrm{sp}}$ for the samples studied. Simultanously, the intensity of the light incident on the sample was measured by a thermocouple: $I_{0}=\lambda V_{\text {th }}$, where $V_{\text {th }}$ is the voltage read from another lock-in connected with the thermocouple. Light intensity was adjusted to produce the same $\Delta V_{\text {sp }}$ value within measured $\lambda$ range by varying the stabilized d.c. voltage applied to the lamp. The measurements of $\alpha=f(\lambda)$ were carried out also. Results are given in the Table. 
TABLE

Optical absorption coefficient of CdMnTe at $300 \mathrm{~K}\left(E_{\mathrm{g}}=1.47 \mathrm{eV}\right)$.

\begin{tabular}{l|l|l|l|l|l|l|l|l|l|l}
\hline \hline$\lambda(\mu \mathrm{m})$ & .810 & .815 & .820 & .825 & .830 & .835 & .840 & .845 & .850 & .855 \\
$\alpha\left(\mathrm{cm}^{-1}\right)$ & 625 & 483 & 400 & 339 & 266 & 193 & 161 & 112 & 104 & 54.8
\end{tabular}

The results of the experiment plotted on the $I_{0}$ versus $\alpha^{-1}$ graph, with $\Delta V_{\mathrm{sp}}$ as a parameter, are shown in Fig. 2. The value of minority carrier diffusion length, read off as a negative intercept of zero light intensity with $\alpha^{-1}$ axis, is equal to $L_{D}=26 \mu \mathrm{m}$, independent of the value of $\Delta V_{\mathrm{sp}}$ adjusted. However increasing departure from linearity with increasing $\Delta V_{\mathrm{sp}}$ for higher value of $\lambda$ is observed. This can be due to the fact that in this case, assumption $l>\alpha^{-1}$ is no longer valid and the surface photovoltage at the back face becomes significant (similar results were reported in [3]).

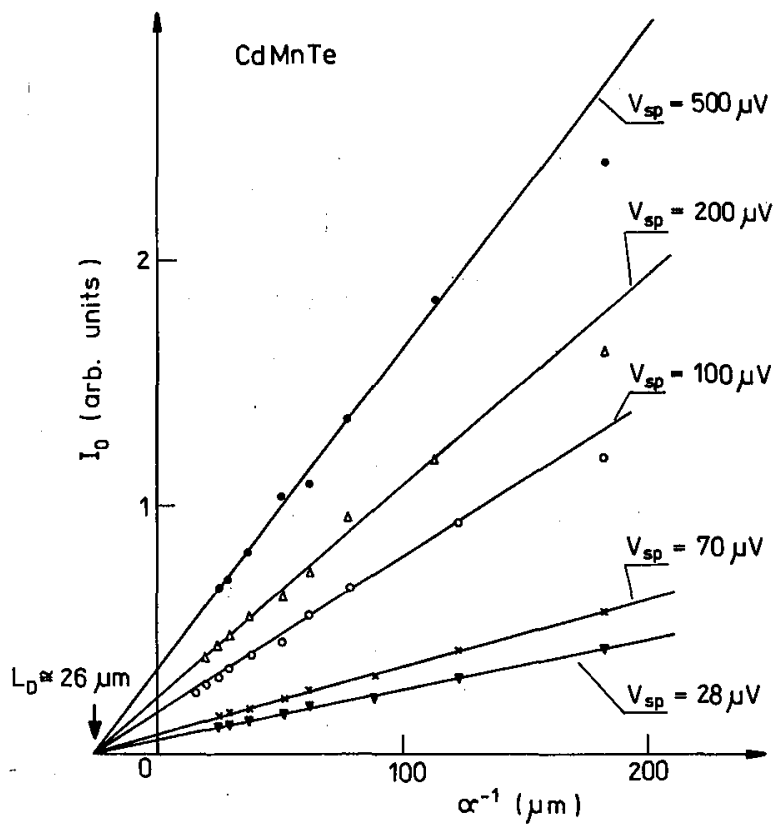

Fig. 2. Light intensity $I_{0}$ versus reciprocal of absorption coefficient $\alpha^{-1}$ with surface photovoltage $\Delta V_{\mathrm{sp}}$ as a parameter for a wide-gap $\left(E_{\mathrm{g}}=1.47 \mathrm{eV}\right.$ at $\left.300 \mathrm{~K}\right) \mathrm{CdMnTe}$. Sample thickness $d=290 \mu \mathrm{m}$.

We also observed that scattered light and decrease of aperture affect the $I_{0}=f\left(\alpha^{-1}\right)$ linearity. In the latter case, the condition of low injection is probably not fulfilled.

In the present paper we have shown the applicability of surface photovoltage method to determine minority carrier diffusion length in wide-gap $\mathrm{CdMnTe}$ 
compound. The value of $L_{D}$ was found to be equal to $26 \mu \mathrm{m}$, for the samples investigated.

\section{References}

[1] U. Emin, M.Sc. Thesis METU, Gaziantep, Turkey (1987).

[2] A.M. Goodman, J. Appl. Phys. 32, 2550 (1961).

[3] M. Saritas, H.D. McKell, Solid-State Electron. 31, 835 (1988). 